\section{FRI0086 CHINA-MANUFACTURED ADALIMUMAB BIOSIMILAR, HLX03, DEMONSTRATED PHARMACOKINETIC EQUIVALENCE AND COMPARABLE SAFETY TO REFERENCE ADALIMUMAB}

Jixun Sun ${ }^{1}$, Guiling Chen ${ }^{1}$, Min Wu', Yiwen $\mathrm{Han}^{2}, \mathrm{He} \mathrm{Gao}^{2}$, Tianli Zhang², Xinjun Guo ${ }^{2}$, Xin Zhang ${ }^{2}$, Eugene Liư ${ }^{2}$, Alvin Luk ${ }^{2}$, Weidong Jiang ${ }^{2}$, Scott Liu ${ }^{2}$ Katherine Chai ${ }^{2}$, Yanhua Ding ${ }^{1}{ }^{1}$ The First Hospital of Jilin University, Jilin, China; ${ }^{2}$ Shanghai Henlius Biotech, Inc., Shanghai, China

Background: Adalimumab first launched in China in August 2010 with now more than 10 million people have its indications. ${ }^{1,2}$ However, the relative high cost of the biologic drug limits the treatment accessibility and reduces the quality of life in patients living with the chronic inflammatory disease like rheumatoid arthritis and psoriasis. In accordance with the China National Medical Product Administration (NMPA) biosimilar regulatory development pathway, biosimilar products require to demonstrate similarity in pharmacokinetics (PK) and safety profile compared to its reference drug, which could further address the unmet medical needs of adalimumab. HLX03 was developed as a proposed biosimilar to adalimumab with the potential to increase affordable treatment options for access.

Objectives: The study was aiming to compare the pharmacokinetics (PK), safety and immunogenicity of the proposed adalimumab biosimilar HLX03 with reference product.

Methods: We conducted a randomised, double-blind, parallel-controlled clinical trial (NCT03357939) in China to compare the PK, safety and immunogenicity of $\mathrm{HLXO3}$ and China sourced adalimumab (CN-adalimumab). In this study, 211 healthy volunteers were randomised 1:1 to receive a single $(40 \mathrm{mg})$ subcutaneous injection of $\mathrm{HLXO3}$ or $\mathrm{CN}$-adalimumab. The primary PK endpoints were area under the curve (AUC) from time zero to the last quantifiable concentration $\left(\mathrm{AUC}_{0-t}\right)$ and maximum observed concentration $\left(\mathrm{C}_{\max }\right)$, secondary endpoint was AUC from time zero to infinity $\left(A \cup C_{0 \text {-inf }}\right.$. PK equivalence was established if the $90 \%$ confidence interval $(\mathrm{Cl})$ for the test-to-reference ratio fall within the 80 $125 \%$ equivalence margin.

Results: Based on the analysis of 210 subjects in the per protocol population (PPS) and 211 subjects in the full analysis set (FAS), HLX03 demonstrated PK equivalence to $\mathrm{CN}$-adalimumab for all primary endpoints (Table 1). The incidents of adverse events (AEs) between two treatment groups were similar, with treatment-emergent AEs (TEAEs) noted by a total of $149(70.0 \%), 79(73.8 \%)$ in HLX03 and $70(66.0 \%)$ in CN-adalimumab group, respectively. One subject suffered non-drug-related severe $\mathrm{AE}$ (tuberculosis) in the $\mathrm{HLX03}$ arm, and one subject occurred grade 4 $\mathrm{AE}$ (elevated creatine phosphokinase) in the $\mathrm{CN}$-adalimumab arm. In the group of $\mathrm{CN}$-adalimumab, 6 more incidents of positive anti-drug antibodies (ADA) recorded at day 7 and no further significant difference observed. Based on the established clinical PK equivalence and safety similarities, 262 patients with moderate-to-severe chronic plaque psoriasis were randomized in 21 centers at 1:1 ratio to conduct a double-blind, parallelcontrolled phase 3 study (NCT03316781) to further evaluate the efficacy and safety profiles of HLX03 and reference adalimumab. The primary efficacy endpoint was the improvement rate of Psoriasis Area and Severity Index (PASI) over the baseline at week 16

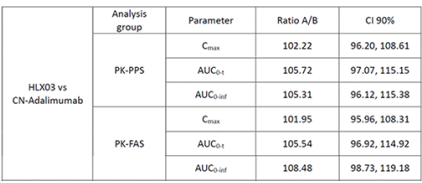

Conclusion: PK equivalence and safety similarities between HLX03 and $\mathrm{CN}$-adalimumab were demonstrated which leads to a multi-center, randomised, double-blind, parallel-controlled phase 3 study to further evaluate the efficacy and safety of $\mathrm{HLXO3}$ as the proposed biosimilar of adalimumab in patients with moderate-to-severe plaque psoriasis.

\section{REFERENCES:}

[1] Chinese Rheumatology Association. 2018 Chinese guideline for the diagnosis and treatment of rheumatoid arthritis[J]. Zhonghua nei ke za zhi, 2018, 57(4): 242

[2] Markets, R. (2018). Investigation Report on China Adalimumab Market, 2010-2018. Retrieved from https://www.prnewswire.com/news-releases/ investigation-report-on-china-adalimumab-market-2010-2018-252895801. $\mathrm{html}$
Disclosure of Interests: Jixun Sun Grant/research support from: research funding, Guiling Chen Grant/research support from: research funding, Min Wu Grant/research support from: research funding, Yiwen Han Employee of: I am an employee of Shanghai Henlius Biotech, Inc., He Gao Employee of: I am an employee of Shanghai Henlius Biotech,Inc., Tianli Zhang Employee of: employee of Shanghai Henlius Biotech, Inc., Xinjun Guo Employee of: Employee of Shanghai Henlius Biotech Inc., Xin Zhang Employee of: I am an employee of Shanghai Henlius Biotech,Inc., Eugene Liu Employee of: I am an employee of Shanghai Henlius Biotech,Inc., Alvin Luk Employee of: I am an employee of Shanghai Henlius Biotech,Inc., Weidong Jiang Shareholder of: I am the co-founder of Shanghai Henlius Biotech,Inc., Employee of: I am an employee of Shanghai Henlius Biotech,Inc., Scott Liu Shareholder of: I am the CEO of Shanghai Henlius Biotech,Inc., Employee of: I am an employee of Shang hai Henlius Biotech,Inc., Katherine Chai Employee of: I am an employee of Shanghai Henlius, Yanhua Ding Grant/research support from: research funding

DOI: 10.1136/annrheumdis-2019-eular.1909

\section{FRI0087 EFFICACY, SAFETY, AND IMMUNOGENICITY RESULTS OF THE SWITCH FROM REFERENCE ADALIMUMAB (REFADL) TO SANDOZ BIOSIMILAR ADALIMUMAB (GP2017, SDZ-ADL) FROM ADMYRA PHASE 3 STUDY IN PATIENTS WITH MODERATE-TO-SEVERE RHEUMATOID ARTHRITIS (RA)}

Piotr Wiland ${ }^{1}$, Sławomir Jeka ${ }^{2}$, Eva Dokoupilova ${ }^{3,4}$, Juan Manuel Miranda Limon ${ }^{5}$, Julia Jauch-Lembach ${ }^{6}$, Anjali Thakur ${ }^{6}$, Halimuniyazi Haliduola ${ }^{6}$, Norman Gaylis ${ }^{7}$. ${ }^{1}$ Wroctaw Medical University, Wroctaw, Poland; ${ }^{2}$ Collegium Medicum UMK, 2nd University Hospital, Bydgoszcz, Poland; ${ }^{3}$ MEDICAL PLUS s.r.o, Uherské Hradiště, Czech Republic; ${ }^{4}$ University of Veterinary and Pharmaceutical sciences, Faculty of Pharmacy, Brno, Czech Republic; ${ }^{5}$ RM Pharma Specialists, Mexico City, Mexico ${ }^{6}$ Hexal AG, Holzkirchen, Germany; ${ }^{7}$ Arthritis and Rheumatic Disease Specialties, Aventura, FL, United States of America

Background: SDZ-ADL is approved by EMA in all indications of refADL based on preclinical and clinical study results. EMA submission data included results of a Phase 3 study in patients with plaque psoriasis. ADMYRA was a Phase 3 study comparing efficacy and safety of SDZ$A D L$ and refADL in patients with moderate-to-severe RA with inadequate response to disease modifying anti-rheumatic drugs, including methotrexate (NCT02744755).

Objectives: The ADMYRA study was designed to compare the efficacy of SDZ-ADL and refADL over 24 weeks of treatment, and to evaluate longterm efficacy, safety, and immunogenicity of SDZ-ADL up to Week (Wk) 48. The study also investigated the effect of the switch from refADL to SDZ-ADL at Wk 24 on efficacy, safety, and immunogenicity up to Wk 48. This abstract focuses on the data after switch from refADL to SDZADL.

Methods: Eligible patients were randomized $1: 1$ to receive $40 \mathrm{mg}$ subcutaneous SDZ-ADL or refADL every other week from Wk 0-22. At Wk 24, patients in the refADL arm were switched to receive SDZ-ADL; treatment continued until Wk 46. The primary endpoint was change in Disease Activity Score-28 including high-sensitivity C-reactive protein (DAS28-CRP) from baseline at Wk 12. Secondary endpoints included mean changes in DAS28-CRP scores, the proportion of patients fulfilling EULAR responses, EULAR and Boolean remission criteria, safety, and immunogenicity. EULAR remission was defined as DAS28-CRP $<2.6$. Boolean remission was defined as tender joint count $28 \leq 1$, swollen joint count $28 \leq 1$, CRP $\leq 1 \mathrm{mg} / \mathrm{dL}$, and patient global assessment $\leq 10$ on a $100 \mathrm{~mm}$ scale.

Results: As reported previously, mean change in DAS28-CRP from baseline at Wk 12 was -2.16 for SDZ-ADL $(\mathrm{N}=140)$ and -2.18 for refADL $(\mathrm{N}=144) .{ }^{1}$ Efficacy in both treatment arms was maintained throughout the study, also after the switch from refADL to SDZ-ADL at Wk 24. Mean change in DAS28-CRP from baseline at Wk 48 was -2.90 and -2.92 for refADL/switched and SDZ-ADL groups, respectively. At Wk 48, the proportion of patients with moderate/good EULAR response was 29.6/68.0\% in refADL/switched group and $29.0 / 69.2 \%$ in SDZ-ADL group. At Wk 48 , the proportion of patients in EULAR remission was 54.4 and $51.4 \%$ and in Boolean remission was 21.6 and $16.8 \%$ for refADL/switched and SDZADL groups, respectively. As previously reported, safety and immunogenicity were similar in both arms before switch. ${ }^{1}$ No new safety concerns were identified after switch. After switch, 32.5\% of patients in the refADL/ switched group and $36.5 \%$ of patients in the SDZ-ADL group experienced adverse events (AEs); serious AEs were reported by 3.6 and $2.5 \%$, respectively. The proportion of patients reporting injection site reactions after switch was $1.2 \%$ in the refADL/switched group and $0.6 \%$ in the SDZ-ADL group, respectively. After switch, 26.3 and $24.0 \%$ of patients 
were positive for antidrug antibodies (ADAs) in refADL/switched and SDZADL groups, respectively. Of these, 81.0 vs $72.2 \%$ were neutralizing. ADA positivity had no clinically meaningful impact on safety.

Conclusion: After the switch from reference to biosimilar, the rates of EULAR remission/response and Boolean remission were high and maintained until Week 48. Treatment switch from refADL to SDZ-ADL at Wk 24 did not impact efficacy, safety, or immunogenicity.

REFERENCE:

[1] Wiland P, et al. Arthritis Rheumatol 2018;70(suppl 10).

Disclosure of Interests: Piotr Wiland Speakers bureau: Novartis, Pfizer, Abbvie, Gedeon-Richter, Lilly, Roche and Sandoz, Sławomir Jeka: None declared, Eva Dokoupilova: None declared, Juan Manuel Miranda Limon: None declared, Julia Jauch-Lembach Employee of: Hexal AG, Anjali Thakur Employee of: Hexal AG, Halimuniyazi Haliduola Employee of: Hexa AG, Norman Gaylis Grant/research support from: Multiple clinical research trials, BMS, AbbVie, GSK, Janssen, Amgen, Pfizer, Regeneron, UCB, Sanofi, SetPoint, ImmunPharma, Astra Zeneca, Sandoz, Novartis, Gilead, Consultant for: electroCore

DOI: 10.1136/annrheumdis-2019-eular.960

\begin{tabular}{|l|l|}
\hline FRI0088 & SAFETY, IMMUNOGENICITY AND EFFICACY OF THE \\
PROPOSED BIOSIMILAR MSB11022 (MODIFIED \\
FORMULATION) COMPARED WITH ADALIMUMAB \\
REFERENCE PRODUCT IN PATIENTS WITH \\
MODERATELY TO SEVERELY ACTIVE RHEUMATOID \\
ARTHRITIS: AURIEL-RA, A RANDOMISED, DOUBLE- \\
BLIND, PHASE III STUDY
\end{tabular}

Christopher Edwards ${ }^{1}$, Veronica Chyrok ${ }^{2}$, Joëlle Monnet ${ }^{2}$, Martin Ullmann ${ }^{2}$, Pantelis Vlachos ${ }^{3} .{ }^{1}$ University Hospital Southampton, Southampton, United Kingdom; ${ }^{2}$ Fresenius Kabi, Eysins, Switzerland; ${ }^{3}$ Cytel, Geneva, Switzerland

Background: Adalimumab is a fully human anti-TNF monoclonal antibody indicated for the treatment of multiple inflammatory disorders, including rheumatoid arthritis (RA). MSB11022 is a proposed adalimumab biosimilar that has been shown to be structurally and functionally similar to the adalimumab reference product ${ }^{1}$. MSB11022 has been developed in two formulations, in a citrate-based buffer, and in a modified buffer and stabiliser. MSB11022 demonstrated bioequivalence and comparable safety, tolerability and immunogenicity profiles to reference adalimumab (both in citrate formulations) in a study in healthy volunteers ${ }^{2}$. Subsequently MSB11022 was shown to be therapeutically equivalent to reference adalimumab (both citrate formulations) in terms of efficacy, safety and immunogenicity in psoriasis patients in the 52-week, Phase III, pivotal AURIEL PsO study ${ }^{3}$. MSB11022 (modified formulation) demonstrated bioequivalence and a comparable safety and immunogenicity profile to MSB11022 (citrate formulation) in an additional healthy volunteer study (EMR200588-003) 4 .

Objectives: To evaluate safety, immunogenicity and efficacy of MSB11022 (modified formulation) in patients with moderately to severe active rheumatoid arthritis compared to reference adalimumab up to 52 weeks.

Methods: RA patients receiving methotrexate were randomised $1: 1$ to MSB11022 (modified formulation) or reference adalimumab (citrate formulation) in the double-blind, multicentre, phase III AURIEL-RA study (NCT03052322). Safety, efficacy and immunogenicity endpoints were assessed at scheduled visits up to week 52 using descriptive statistical methods only. Safety was the primary objective and the study was not powered to demonstrate equivalent efficacy.

Results: 288 RA patients were randomised (MSB11022, $n=143$; reference adalimumab, $\mathrm{n}=145$ ). Patient baseline characteristics were comparable between treatment groups. Few adverse events of special interest (AESIs) of hypersensitivity (primary endpoint) were reported during the study and the proportions were similar across treatment arms. Efficacy endpoints, including the key secondary endpoint of American College of Rheumatology criteria 20\% (ACR20) response rate at week 12, were similar between the treatment arms. The safety profiles of patients receiving MSB11022 and reference adalimumab were also similar through to week 52. There were no clinically meaningful differences in the incidence of anti-drug antibodies (ADAs) and neutralising antibodies (NAbs) between treatment arms up to week 52. Key results are presented in Table 1. Conclusion: MSB11022 (modified formulation) and reference adalimumab had similar safety, immunogenicity and efficacy profiles over 52 weeks in patients with RA, supplementing the clinical data collected with MSB11022 (citrate formulation) in healthy volunteers and psoriasis patients
Table 1

\begin{tabular}{lccc}
\hline Endpoint (analysis set) & $\begin{array}{c}\text { Timepoint } \\
\text { (Week) }\end{array}$ & MSB11022 & $\begin{array}{c}\text { Reference } \\
\text { adalimumab }\end{array}$ \\
\hline Incidence of AESIs of hypersensitivity & 52 & $6(4.2)$ & $8(5.5)$ \\
(SAF) & 52 & $83(58.0)$ & $93(64.1)$ \\
Incidence of TEAEs (SAF) & 12 & $113(79.6)$ & $114(80.9)$ \\
ACR20 response (ITT) & 12 & $3.3 \pm 1.2$ & $3.4 \pm 1.2$ \\
DAS28-ESR (ITT) & 52 & $115(80.4)$ & $104(71.7)$ \\
Overall incidence of ADAs (SAF) & 52 & $57(39.9)$ & $57(39.3)$ \\
Overall incidence of NAbs (SAF) & Numbers are $n$ (\%) or mean \pm standard deviation. DAS28, Disease Activity Score-28; ESR, \\
erythrocyte sedimentation rate; ITT, intention-to-treat set; SAF, safety analysis set; TEAE, \\
treatment-emergent adverse event
\end{tabular}

REFERENCES:

[1] Magnenat L, et al. MAbs 2017:9(1):127-39.

[2] Hyland E, et al. Br J Clin Pharmacol 2016;82(4):983-93.

[3] Hercogova J, et al. J Am Acad Dermatol 2018;79(3):AB21.

[4] Fresenius Kabi. Data on File 2019

Disclosure of Interests: Christopher Edwards Grant/research support from Abbvie, BMS, Biogen, Celgene, Fresenius, Janssen, Lilly, Mundipharma, Pfizer, MSD, Novartis, Roche, Samsung, Sanofi, UCB, Consultant for: Abbvie, BMS, Biogen, Celgene, Fresenius, Janssen, Lilly, Mundipharma, Pfizer, MSD, Novartis, Roche, Samsung, Sanofi, UCB, Speakers bureau: Abbvie, BMS, Biogen, Celgene, Fresenius, Janssen, Lilly, Mundipharma, Pfizer, MSD, Novartis, Roche, Samsung, Sanofi, UCB, Veronica Chyrok Employee of: Former employee of Fresenius Kabi SwissBioSim

Joëlle Monnet Employee of: Employee of Fresenius Kabi SwissBioSim, Martin Ullmann Shareholder of: Amgen, BMS, Employee of: Employee of Fresenius Kabi SwissBioSim, Pantelis Vlachos: None declared DOI: 10.1136/annrheumdis-2019-eular.4220

\section{\begin{tabular}{|l|l}
\hline FRI0089 SAFETY AND EFFICACY OF RADIONUCLIDE \\
\hline
\end{tabular} SYNOVECTOMY IN PATIENTS WITH PERSISTENT INFLAMMATORY OF SINGLE JOINT IN THE COURSE OF BIOLOGICAL THERAPY}

Anna Felis-Giemza ${ }^{1}$, Marek Chojnowski ${ }^{2}$, Julita Rejmer ${ }^{1}$, Leszek Królicki ${ }^{2}$, Marzena Olesińska ${ }^{1} .{ }^{1}$ National Institute of Geriatrics, Rheumatology and Rehabilitation, Connective Tissue Diseases Department, Warsaw, Poland; ${ }^{2}$ Public Central Teaching Hospital, Nuclear Medicine Department, Warsaw, Poland

Background: Radionuclide synovectomy (RSV) is a form of minimally invasive treatment of persistent joint inflammation. Primary indication for RSV is hypertrophic synovitis refractory to disease-modifying anti-rheumatic drugs (DMARDs), whether synthetic or biological, and intraarticular steroid injections. This procedure has a high rate of success with a low rate of adverse events and complications in properly selected patients. Moreover, due to bactericidal properties of the radiocolloids, the risk of infection after RSV is insignificant, and incidence of post-injection septic arthritis is extremely low.

Objectives: To assess safety and efficacy of RSV in patients with inflammatory rheumatic diseases treated with biological disease-modifying antirheumatic drugs (bDMARDs).

Methods: We analyzed outcomes of 76 radionuclide synovectomy interventions in 47 patients (37 female and 10 male) ongoing biological therapy. The patients were diagnosed as follows: rheumatoid arthritis (RA) 37, ankylosing spondylitis (AS) -7 , psoriatic arthritis (PsA) -2 and juvenile idiopathic arthritis (JIA) -1 patient. The majority of the patients were treated with TNF-alpha inhibitors $(79 \%)$, which included: adalimumab (31.6\%), etanercept $(25 \%)$, golimumab $(14.5 \%)$ and certolizumab $(7.9 \%)$. The other patients were treated with interleukin-6 receptor antagonist tocilizumab (19,7\%) and anti-CD20 monoclonal antibody rituximab (1.3\%). Patients with overall good response to biologics and persistent inflammation of single joint confirmed by ultrasound PD examination, in case of no contraindications were qualified for RSV procedure. For the RSV following radiopharmaceuticals were used: rhenium-186 sulphide in 41 cases for shoulder (2), elbow (13), wrist (22), hip (2) and ankle (2) joints; ytrium-90 citrate in 27 cases for knee joints; erbium-169 citrate in 8 cases for small joints of hand and feet. All patients had a follow-up visit 3 and 6 months after RSV, during which a clinical and ultrasound examination of the treated joints were performed. Continuous adverse events collection was conducted.

Results: The most common indication for RSV was RA - 60 procedures (78.9\%), followed by AS - 10 (13.2\%), PsA - 4 (5.3\%) and JIA - 1 procedure $(2.6 \%) .27$ patients had RSV intervention in one joint. 17 